\title{
Assessment of self-consistent field convergence in spin-dependent relativistic calculations
}

\author{
Masahiko Nakano ${ }^{\mathrm{a}}$, JunjiSeino ${ }^{\mathrm{b}}$, and Hiromi Nakai ${ }^{\mathrm{a}-\mathrm{d}, *}$ \\ ${ }^{\mathrm{a}}$ Department of Chemistry and Biochemistry, School of Advanced Science and \\ Engineering, Waseda University, Tokyo 169-8555, Japan \\ ${ }^{\mathrm{b}}$ Research Institute for Science and Engineering, Waseda University, Tokyo 169-8555, \\ Japan \\ 'CREST, Japan Science and Technology Agency, Kawaguchi 332-0012, Japan \\ ${ }^{\mathrm{d}}$ ESICB, Kyoto University, Kyoto 615-8520, Japan
}

*Corresponding author. Phone: +81-3-5286-3452.Fax: +81-3-3205-2504.

E-mail address: nakai@waseda.jp (H. Nakai).

(C) 2016. This manuscript version is made available under the Elsevier user license http://www.elsevier.com/open-access/userlicense/1.0/ 


\begin{abstract}
This Letter assesses the self-consistent field (SCF) convergence behavior in the generalized Hartree-Fock (GHF) method. Four acceleration algorithms were implemented for efficient SCF convergence in the GHF method: the damping algorithm, the conventional direct inversion in the iterative subspace (DIIS), the energy-DIIS (EDIIS), and a combination of DIIS and EDIIS. Four different systems with varying complexity were used to investigate the SCF convergence using these algorithms, ranging from atomic systems to metal complexes.The numerical assessments demonstrated the effectiveness of a combination of DIIS and EDIIS for GHF calculations in comparison with the other discussed algorithms.
\end{abstract}




\section{Introduction}

In chemistry and physics, relativistic effects are vital to accurately describe the heavier elements. These effects are classified into two primary types: spin-free (SF) or scalar relativistic effects, which are mainly responsible for orbital contraction and expansion; and spin-dependent (SD) effects, which induce energy level splitting through the coupling of orbital and spinangular momenta. In quantum chemical calculations, SF

effectsare included by perturbative treatments or by using the same ansatz as a non-relativistic (NR) treatment. SD effectscan be considered by using either the spin-orbit configuration interaction (SOCI) method or perturbative treatments[1,2]. These schemes for the SD effects are effective for light elements, whose relativistic effects are comparatively small, and the SD effectsof these systems can be treated as an additional correction to the NR or SF relativistic calculations. Alternative approaches to include SD effects are the two-and four-component relativistic methods[3-17]. These treatments give accurate results across the wholeperiodic table because the relativistic effects are explicitly considered in the self-consistent field (SCF) calculations.

In SD calculations, generally, the spin symmetries of the two- and four-component relativistic wavefunctions are broken because spin is not a good quantum number. To describe the correct spin behavior, the generalized Hartree-Fock (GHF) method[18-25], 
where any symmetry constraints are removed, can be used instead of either the restricted HF (RHF) or unrestricted HF (UHF). Because the additional spin degrees of freedom rotate the spin-quantized axes independently, GHF is also termed a non-collinear method.

However, it is well-known that the convergence in GHF calculations is difficult due to the additional spin degrees of freedom. This sometimes causes the calculations to fall into a higher energy saddle point. One solution for the local minima problem is an extension of the second-order orbital optimization scheme to GHF, which has been proposed by Goings et al. [26].In this study, we tackle the convergence problem in the GHF calculations from the viewpoint of the SCF acceleration techniques.Here, we implement four techniques to GHF, which are typical for NR calculations. The firstmethod is the use of a damping algorithm, the simplest form of acceleration algorithm. The second, and most popular,method isPulay's direct inversion in the iterative subspace (DIIS) method[27,28]. A number of variants of the DIIS algorithm have been developed, and these also accelerate SCF convergence [29-33]. One DIIS variant, the energy-DIIS (EDIIS) developed by Kudin et al.[29], is also assessed in this study. The fourth scheme assessed here is a combination algorithm comprising the DIIS and EDIIS algorithms, and termed EDIIS+DIIS. This algorithm was assessed by Garza 
and Scuseria[34], and Sulzer et al. [33], and they concluded that this combination algorithm is the best choice for NR molecular calculations.

This Letter is organized as follows: Section 2 presents, briefly, theoretical aspects used in this study. Then, the numerical assessments are shown and discussed in Sec. 3, and the concluding remarks are given in Sec. 4. 


\section{Theoretical aspects}

This section provides brief explanations of GHF theory and the SCF acceleration algorithms in the GHF framework.In the GHF method, molecular spinors (MSs), which areeigenfunctions of the Fock matrix, are defined by the superposition of atomic orbitals (AOs) for alpha- and beta-spins, i.e.,

$$
\varphi_{i}=\sum_{\sigma}^{\{\alpha, \beta\}} \sum_{\mu} C_{\mu i}^{\sigma} \chi_{\mu}^{\sigma} \sigma
$$

where $\varphi$ denotes the MSs, $\chi$ denotes the AOs, $C$ denotes the spinor coefficients, and $\sigma$ denotes the spin functions. In the two-component framework, the Roothaan-Hall (RH) equation is expressed in block form,

$$
\left(\begin{array}{ll}
\mathbf{F}^{\alpha \alpha} & \mathbf{F}^{\alpha \beta} \\
\mathbf{F}^{\beta \alpha} & \mathbf{F}^{\beta \beta}
\end{array}\right)\left(\begin{array}{l}
\mathbf{C}^{\alpha} \\
\mathbf{C}^{\beta}
\end{array}\right)=\left(\begin{array}{cc}
\mathbf{S}^{\alpha \alpha} & \mathbf{0} \\
\mathbf{0} & \mathbf{S}^{\beta \beta}
\end{array}\right)\left(\begin{array}{l}
\mathbf{C}^{\alpha} \\
\mathbf{C}^{\beta}
\end{array}\right) \boldsymbol{\varepsilon},
$$

where $\mathbf{F}$ is the Fock matrix, $\mathbf{S}$ is the overlap matrix, and $\boldsymbol{\varepsilon}$ is the spinor energy. The density matrix in GHF also has the block form,

$$
\mathbf{D}=\left(\begin{array}{ll}
\mathbf{D}^{\alpha \alpha} & \mathbf{D}^{\alpha \beta} \\
\mathbf{D}^{\beta \alpha} & \mathbf{D}^{\beta \beta}
\end{array}\right)=\left(\begin{array}{l}
\mathbf{C}^{\alpha} \\
\mathbf{C}^{\beta}
\end{array}\right) \mathbf{n}\left(\begin{array}{l}
\mathbf{C}^{\alpha} \\
\mathbf{C}^{\beta}
\end{array}\right)^{\dagger},
$$

where $\mathbf{n}$ denotes the occupation numbers.

Here, we use five techniques to solve the RH equation. The first is the fixed-point (FP) algorithm, which uses no acceleration techniques. The second is the static damping algorithm, whose equation is written as 


$$
\mathbf{D}^{\text {new }}=\kappa \mathbf{D}_{i-1}+(1-\kappa) \mathbf{D}_{i}
$$

where $\mathbf{D}_{i}$ denotes the density matrix in the $i$-th iteration and $\kappa$ is the weighting factor. In the NRcalculations, the damping algorithm is stable, but its rate of convergence is slow.

The other techniques we have used are related to the DIIS algorithm. In these algorithms, a new density matrix is estimated by the linear combination of the density matrices from the previous SCF iterations,

$$
\mathbf{D}=\sum_{i=1}^{n} c_{i} \mathbf{D}_{i}
$$

where $n$ is the number of the dimension of the DIIS subspace.This treatment is also available for the Fock matrix instead of the density matrix because of the linear relationship between the density and Fock matrices. The third techniques used here is the conventional DIIS method, which optimizes the coefficients $\left\{c_{i}\right\}$ by minimizing the so-called DIIS error vector e. The error vector is commonly given by $\mathbf{e}=[\mathbf{F}, \mathbf{D}]=\mathbf{F D}-\mathbf{D F}$ in an orthonormal basis. This is because $[\mathbf{F}, \mathbf{D}]=\mathbf{0}$ is the necessary condition for a converged SCF.The optimal DIIS coefficients aremathematically given by

$$
\left\{c_{i}\right\}=\arg \inf \left\{\left\langle\sum_{j=1}^{n} c_{j} \mathbf{e}_{j} \mid \sum_{k=1}^{n} c_{k} \mathbf{e}_{k}\right\rangle, \quad \sum_{i=1}^{n} c_{i}=1\right\} .
$$

Here, the working equation to obtain the coefficientsis written as 


$$
\left(\begin{array}{cc}
\mathbf{B} & \mathbf{1}^{\mathrm{t}} \\
\mathbf{1} & 0
\end{array}\right)\left(\begin{array}{l}
\mathbf{c} \\
\lambda
\end{array}\right)=\left(\begin{array}{l}
\mathbf{0} \\
1
\end{array}\right)
$$

where

$$
\begin{aligned}
& B_{i j}=\left\langle\mathbf{e}_{i} \mid \mathbf{e}_{j}\right\rangle, \\
& \mathbf{c}_{i}=\left(\begin{array}{llll}
c_{1}, & c_{2}, & \cdots, & c_{n}
\end{array}\right)^{\mathrm{t}}, \\
& \mathbf{1}=(1, \quad 1, \cdots, 1)^{\mathrm{t}} .
\end{aligned}
$$

Here, $\lambda$ is a Lagrange multiplier. Equation(7)isa linear equation and is solved by matrix inversion.In the NR calculations, DIIS performance is known to depend on the initial guess, although the rate of convergence is fast. In the RHF/UHF calculations for the relativistic Hamiltonian including only SF terms, denoted as SF-RHF/UHF, Eq. (7) can be straightforwardly applied. On the other hand, in the GHF calculations for the relativistic Hamiltonian involving not only SF terms but also SD ones, denoted as SD-GHF, the Fock and density matricesbecome complex and have dimensions twice the size of those of NR and SF relativistic calculations. Thus, Eq. (7) is solved in complex space.

The fourth technique is the EDIIS method. The coefficients for the linear combination of the previously iterated density matricesis given by

$$
\left\{c_{i}\right\}=\operatorname{arginf}\left\{E^{\mathrm{HF}}\left(\sum_{i=1}^{n} c_{i} \mathbf{D}_{i}\right), \quad \sum_{i=1}^{n} c_{i}=1, \quad c_{i} \in[0,1]\right\},
$$


where $E^{\mathrm{HF}}$ is the HF energy functional, which is defined as,

$$
E^{\mathrm{HF}}\left(\sum_{i=1}^{n} c_{i} \mathbf{D}_{i}\right)=\sum_{i=1}^{n} c_{i} E^{\mathrm{HF}}\left(\mathbf{D}_{i}\right)-\frac{1}{4} \sum_{i=1}^{n} \sum_{j=1}^{n} c_{i} c_{j}\left\langle\mathbf{D}_{i}-\mathbf{D}_{j} \mid \mathbf{F}_{i}-\mathbf{F}_{j}\right\rangle .
$$

This means that the EDIIS coefficients are chosen to minimize the HF energy functional.The minimization problemunder the restriction of $c_{i} \in[0,1]$ issolved byconstrained optimization methods such as the reduced gradient algorithm[35].EDIIS is known to work efficiently even if the SCF calculation starts from poor initial guess orbitals. However, the rate of convergence is slower near the minimum than that of DIIS. The imaginary part of the second term of Eq. (12)is normally approximated to be zero even in complex SD-GHF calculations.Thus, for minimization, we have used real coefficients.

The final and fifth technique is a combination algorithm comprising DIIS and EDIIS, known as EDIIS+DIIS. As described before, EDIIS is efficient even when starting with a poor initial guess and DIIS is efficient near the minimum. Thus, a combination algorithmis more efficientthan either of the algorithms separately. Here, we use a similar EDIIS+DIIS algorithm to that used in a previous study of NR calculations[34].In the early steps, EDIIS is used alone, until the largest absolute element of the DIIS error vector is less than $10^{-1}$ a.u. In the region where the largest absolute element is between $10^{-1}$ and $10^{-4}$ a.u., the coefficients for the linear 
combination of the density matrices can be given by

$$
\mathbf{c}=10 \max \left\{e_{p}^{n}\right\} \mathbf{c}^{\mathrm{EDIIS}}+\left(1-10 \max \left\{e_{p}^{n}\right\}\right) \mathbf{c}^{\mathrm{DIIS}},
$$

where $\mathbf{c}^{\text {EDIIS }}$ and $\mathbf{c}^{\text {DIIS }}$ are the EDIIS and DIIS coefficients, respectively. Here $\max \left\{e_{p}^{n}\right\}$ denotes the largest element of the DIIS error vector in the present (n-th) iteration. Finally, in the region where $\max \left\{e_{p}^{n}\right\}$ is less than $10^{-4}$ a.u., DIIS is used alone until SCF convergence is achieved. 


\section{Computational details}

This section describes the computational details usedto assess the acceleration techniques in GHF. We implemented the three DIIS-related algorithms (DIIS, EDIIS, and EDIIS+DIIS) in our in-house program. For comparison, the simple FP and damping algorithms were also used. In the damping algorithm, the weighting factor for the previous density matrix was fixed to $\kappa=0.25$. The maximum number of the dimension of the DIIS and EDIIS subspaces, i.e., the number of the density and Fock matrices involved in the linear combination, was fixed to 20 . The efficiencies of the five algorithms were numerically assessed through SD-GHF level calculations. For comparison of the SCF convergence behavior, SF-RHF/UHF calculations, generally giving better convergence behavior than SD-GHF, were also performed. It should be noted that SF-RHF/UHF methods generally cannot describe spin-orbit interactions, stable spin states of non-collinear spin systems, and so forth.For the relativistic Hamiltonian, the infinite-order Douglas-Kroll-Hess (IODKH) method [13] with an NR two-electron Coulomb operator was adopted. The SD-GHF calculations with the IODKH Hamiltonian include spin-orbit interactions, while the SF-RHF/UHF ones do not.The basis sets used were DKH3-Gen-TK/NOSec-V-TZP [36,37], whose contraction coefficients were optimized with the third-order DKH Hamiltonian. The SCF 
calculations started from the diagonalization of the bare nucleus Hamiltonian and the superposition of the atomic densities guess [38] for atomic and molecular systems, respectively. SCF convergence criteria are the total energy difference of less than $10^{-9}$ a.u. and a maximum absolute difference of the density matrix elements of $10^{-5}$ a.u. from the previous iteration.

Numerical tests were performed for four types of benchmark systems, i.e., neutral atomic systems from $\mathrm{He}$ to $\mathrm{Lr}, \mathrm{W}(\mathrm{CO})_{6}, \mathrm{Cr}_{3}$, and $\mathrm{UF}_{4}$, to investigate the convergence behaviorsof the different acceleration techniques. The geometry of the octahedral $\mathrm{W}(\mathrm{CO})_{6}$ complex was taken from Ref. [39], where $R_{\mathrm{W}-\mathrm{C}}=2.06 \AA$ and $R_{\mathrm{C}-\mathrm{O}}=1.17$ A.The bondlengths of the equilateral triangle $\mathrm{Cr}_{3}$ and tetrahedral $\mathrm{UF}_{4}$ were 2.89 and 2.00 $\AA$, respectively[26,29]. The neutral atomic systems were selected as a test set with various electron configurations from singlet to nonet and with several magnitudes of relativistic effects. The $\mathrm{W}(\mathrm{CO})_{6}$ complex was chosen as a simple example of a heavy-element system whose SCF convergence is well-behaved. $\mathrm{The}^{\mathrm{Cr}_{3}}$ molecule was selected as an example of a non-collinear spin system. Finally, $\mathrm{UF}_{4}$ was chosen as an example of a system for which SCF convergence is difficult to achieve both in the NR and relativistic frameworks. 


\section{Results and discussion}

\subsection{Atomic systems from $\mathrm{He}$ to $\mathrm{Lr}$}

This subsection discusses the SCF convergence behaviors for the atomic systems from He to Lr. The number of cycles required to converge the SCF procedures are summarized for s- and p-block elements in Table 1, d-block elements in Table 2, and f-block elements in Table 3. If the calculations did not converge within 1000 cycles, N.C. is given instead of the number of cycles in the tables. Atomic numbers $(Z)$ and spin multiplicities for the SF calculations $(2 S+1)$ are also shown. The five algorithms, i.e., FP, damping, DIIS, EDIIS, and EDIIS+DIIS, were used for the SD-GHF calculations as well as SF-RHF/UHF ones.

For the s- and p-block elements listed in Table 1, most SF calculations converged; however, convergence with the FP algorithm was not achieved for Ga, Ge, and As. For systems that did converge with the FP algorithm, the number of SCF cycles required ranged from 7 to 32 . In contrast, the damping algorithm required more SCF cycles than FP, but all the calculations using damping converged. In most cases, in terms of the number of cycles, EDIIS was the algorithm most similar to FP. This is because the local search ability of EDIIS is similar to that of FP, although the global search ability is higher in EDIIS. DIIS and EDIIS+DIIS both achieved convergence in the least 
numberof cycles. These results indicate that the local search ability is important for efficient SF calculations in these systems. In the case of the SD calculations, the situation is slightly different from the SF calculations. In FP, several elements required more cycles to achieve convergence: five elements did not converge and 11 elements required more than 100 cycles. In the damping algorithm, the trend is similar to the aboveone in FP, althoughthe algorithm required more cycles than FP for most elements.In the DIIS-based algorithms (DIIS, EDIIS, and EDIIS+DIIS), closed-shell elements with singlet spin, alkaline metals with doublet spin, and carbon group elements with triplet spinexcept Sirequired the similar number of iterations to those of the SF calculations. In contrast, elements with doublet spin states, such as halogens, some triplet spin elements, and quartet spin elements required more cycles compared with the SF calculations.

For the d-block elements listed in Table 2, more cycles were requiredin comparison with the s- and p-block elements. Furthermore, most SF and SD calculations using FP did not converge. At the SF level, DIIS and EDIIS+DIIS required the least number of cycles, as was the case with the s-and p-block elements. At the SD level, EDIIS+DIIS achieved convergence in all cases except $\mathrm{Ru}$. Let us compare the unfavorable convergence behavior of $\mathrm{Ru}$ in comparison with $\mathrm{Fe}$ and $\mathrm{Os}$ in the group 8. The 
electronic configurations of $\mathrm{Ru}, \mathrm{Fe}$, and $\mathrm{Os}$ in the ground states are $[\mathrm{Kr}](4 \mathrm{~d})^{7}(5 \mathrm{~s})^{1}$, $[\mathrm{Ar}](3 \mathrm{~d})^{6}(4 \mathrm{~s})^{2}$, and $[\mathrm{Xe}](4 \mathrm{f})^{14}(5 \mathrm{~d})^{6}(6 \mathrm{~s})^{2}$, respectively. Thus, in the SCF iterations of the SD-GHF calculations for $\mathrm{Ru}$, electronic fluctuation between $4 \mathrm{~d}$ - and 5 s-orbitals might happen, whereas those for Fe and Os might not.

DIIS required less cycles than EDIIS+DIIS in 10 elements including some triplet and quartet spin systems:Sc, Ti, V, Ni, Zn, Zr, Lu, Hf, Ta, and Pt. This behavior may be caused by the feature of DIIS, which is efficient when starting with a good initial guess.

In general, the singlet elements demonstrated a good convergence behavior.In addition, Cr, Mn, Mo, Tc, and Re, which have completely singly-occupied d-orbitals, i.e., $d^{5} s^{1}$ or $d^{5} s^{2}$, and correspond to sextet or septet systems, showed a reasonableconvergence behavior. For these elements, the electronic fluctuation among the d-orbitals is expected to be small, which is the origin of less SCF cycles. The rest elements possess both singly- and doubly-occupied (unoccupied) d-orbitals, corresponding to the doublet, triplet, quintet, and quartet states. Since the d-electron fluctuationmight occur, the SCF convergence became comparatively slowfor these elements.

For the f-block elements listed in Table 3, the convergence behaviors were worse in comparison with elements from other blocks. At the SF level, SCF convergence was not 
achieved using FP, except for Th. In the SF calculations, the SCF calculations for 16 elements failed to converge when using damping, eight for DIIS, five for EDIIS, and one for EDIIS+DIIS. In the SD calculations, the numbers of systems that did not converge were 18 for damping, 11 for DIIS, six for EDIIS, and seven for EDIIS+DIIS. In these systems, global search is important. We did not find a general trend in terms of the SCF convergence for the f-block elements, and the poor convergence behavior of the f-block elements is due to the large SD effects that arise fromopen-shell electrons in the $\mathrm{d}$ - and f-orbitals, which have large angular momenta, from the viewpoint of the jj-coupling scheme.

Table 4 summarizes the statistical results. \#Failure is the sum of the number of elements thatfailed to converge for each algorithm. The average, minimum, and maximum numbers of iterations for the elements that achieved convergence by each method areshown relative to the EDIIS+DIISvalues. They are represented as $\%$ Average, $\%$ Best, and $\%$ Worst, respectively. For example, the $\%$ Average for FP is calculated as

$$
\% \text { Average }(\mathrm{FP})=\frac{\# \text { Average }(\mathrm{FP})-\# \text { Average }(\text { EDIIS }+ \text { DIIS })}{\# \text { Average }(\text { EDIIS }+ \text { DIIS })} \times 100
$$

where\#Average $(X)$ denotes the average number of iterations using algorithm $\mathrm{X}$.

For every algorithm, \#Failure was lowest for $\mathrm{s} / \mathrm{p}$ - and highest for f-block 
elements.Furthermore, the SD calculations had a higher rate of failure(higher \#Failure)than the corresponding SF calculations. In most cases, \#Failure decreased in the order of FP, damping, DIIS, EDIIS, and EDIIS+DIIS, as listed in the table.Taking into account the calculated values of \%Average, \%Best, and \%Worst, in most cases,FP and damping required more cycles than the DIIS-related algorithms. For the DIIS-related algorithms, EDIIS required the most cycles. The performance of DIIS was comparatively similar to that of EDIIS+DIIS. In conclusion, EDIIS+DIIS stably achievesSCF convergence with fewer cycles for both SD and SF relativistic calculations.

\subsection{A well-behaved system: $W(C O)_{6}$}

This subsection discusses the SCF convergence behavior of the $\mathrm{W}(\mathrm{CO})_{6}$ complex.

The complex was selected as an example of a simple heavy-element systemwhose convergence is well-behaved. This is true because $\mathrm{W}(\mathrm{CO})_{6}$ has a completely closed-shell configuration, even in SD calculations. Figure 1 shows the SCF convergence behaviors in GHF for the $\mathrm{W}(\mathrm{CO})_{6}$ complex using $\mathrm{FP}$ and the four acceleration techniques: damping, DIIS, EDIIS, and EDIIS+DIIS. The SF result using EDIIS+DIIS is also shown for comparison. The vertical and horizontal axes showthe 
absolute energy difference between the successive iterations on a logarithmic scale andthe iteration number, respectively.With all algorithms except FP, SCF convergence was achieved. These methods converged to the same total energy $(E=-16809.23228$ a.u.). DIIS and EDIIS+DIIS were the fastest algorithms, converging in the same number of iterations, i.e., 19 cycles. In contrast, EDIIS and damping required more cycles; 39 cycles for EDIIS and 79 cycles for damping. These indicate thatthe initial guess was a good approximate solution for the simple and typical electronic structure of $\mathrm{W}(\mathrm{CO})_{6}$. Thus, the local search performance mainly determines the rate of convergence here.The difference in the number of iterations between the SF and SD calculations was small:14 cycles for EDIIS+DIIS at the SF level. The well-behaved nature of the SCF convergence of $\mathrm{W}(\mathrm{CO})_{6}$ is due to itstypical closed-shell character and small contributions of SD effects to SCF convergence.

\subsection{A non-collinear system: $\mathrm{Cr}_{3}$}

This subsection discusses the SCF convergence behavior of the $\mathrm{Cr}_{3}$ molecule. $\mathrm{Cr}_{3}$ is an example of a non-collinear spin system. In non-collinear systems such as the open-shell triangular $\mathrm{Cr}_{3}$ complex, the stable spin structureis isotropic with respect to the molecular plane due to geometrical frustration. To describe the stable spin structure of 
the non-collinear system, GHF, which freely rotates each spin-quantized axis, is required. An SF-UHF calculation was also performed for comparison of the SCF convergence behavior. Figure 2 shows the SCF convergence results obtained from the GHF calculations for the non-collinear $\mathrm{Cr}_{3}$ system using the four acceleration techniques and the FP algorithm as well as the result of an SF calculation.Only the EDIIS+DIIS algorithm achieved SCF convergence within 1000 cycles. The converged total energy obtained using EDIIS+DIIS in GHF was -3148.60769 a.u. Using FP led to large energy differences, which are due to the oscillation between two states. In damping and DIIS, the energy differences decreased slowly, although they failed to converge within 1000 cycles. In EDIIS, the energy differences also decreased slowly in bothearly and late iterations, as shown in Figure 2. However, in the middle iteration region, the differencesincreased. This result indicates that local searching isimportantin achieving convergence for this system. The difference between SF and SD calculations was larger than that of $\mathrm{W}(\mathrm{CO})_{6}$ because the electronic structure obtained by GHF is more complicated, arising from the geometrical non-collinearity in $\mathrm{Cr}_{3}$. In conclusion, the EDIIS+DIIS approach is effective for non-collinear spin systems.

\subsection{A challenging system: $U F_{4}$}


This subsection describes the SCF convergence behavior in $\mathrm{UF}_{4}$, which is arepresentative challenging case. This system has beenused as a challenging example in previous studies investigating convergence acceleration techniques [29,33,34]. Figure 3 shows the convergence behaviors in the GHF calculations for the $\mathrm{UF}_{4}$ molecule using the FP algorithm and the four acceleration techniques as well as the SF result. In this system, when DIIS and EDIIS+DIIS were used, the SCF calculations converged within 1000cycles. EDIIS+DIIS converged twice as fast as DIIS, although EDIIS+DIIS required 334 iterations to achieve convergence. This result indicates that SCF convergence is challenging in $\mathrm{SD}$ calculations of $\mathrm{UF}_{4}$. The converged total energy was -28380.18867a.u. for EDIIS+DIIS and -28380.18851 a.u. for DIIS. This means that these two calculations converged to different SCF solutions with each other. From the analysis of the converged density matrices, the major differences were observed for 5f-orbitals of the uranium atom, reflecting theirvarious microstates.Damping and EDIIS slowly but monotonically decreased the energy errors, which steadily approached the values obtained by EDIIS+DIIS/DIIS. The difference between the number of iterations required by SF and SD calculations was approximately 200 . This result indicates that spin-orbit interactions contribute significantly to the electronic structure of $\mathrm{UF}_{4}$. Thus, the SCF convergence for $\mathrm{UF}_{4}$ is more difficult at SD than SF level. However, 
EDIIS+DIIS was an effective algorithm for this challenging system.

In summary, the EDIIS+DIIS algorithm is applicable for many cases: typical and simple systems, non-collinear systems, whichrequire GHF treatment to describe the geometrically frustrated spinstructure, and challenging systems, which require GHF treatment with SD effects. 


\section{Conclusion}

In this study, we have assessed theFP algorithm and four SCF accelerationalgorithms including the damping, DIIS, EDIIS, and EDIIS+DIIS algorithms in the complex GHF framework both with SF and SD relativistic effects. The IODKH method with NR two-electron Coulomb interactions was used as a many-electron Hamiltonian. As benchmark systems, we used atomic systems from He to $\mathrm{Lr}$, the $\mathrm{W}(\mathrm{CO})_{6}$ complex as an example of a well-behaved system, $\mathrm{Cr}_{3}$ as a non-collinear spin system, and $\mathrm{UF}_{4}$ as a challenging system. The numerical assessments revealed that the EDIIS+DIIS algorithm provides fast and stable SCF convergence in the GHF framework in comparison with the other algorithms. 


\section{Acknowledgements}

Some of the present calculations were performed at the Research Center for Computational Science (RCCS), the Okazaki Research Facilities, and the National Institutes of Natural Sciences (NINS). This study was supported in part by the Ministry of Education, Culture, Sports, Scienceand Technology (MEXT) program 'Elements Strategy Initiative to Form Core Research Center' (since 2012), and by Core Research for Evolutional Science and Technology (CREST) Program 'Theoretical Design of Materials with Innovative Functions Basedon Relativistic Electronic Theory' of the Japan Science and TechnologyAgency (JST). M.N. is grateful to the Early Bird Program from Waseda Research Institute for Science and Engineering for a Research Grant for Young Scientists. 


\section{References}

[1] K.G. Dyall, K. Faegri, Introduction to Relativistic Quantum Chemistry, Oxford University Press, New York, 2007.

[2] P. Schwerdtfeger, Relativistic Electronic Structure Theory, Part 2. Applications, Elsevier Science, Amsterdam, 2004.

[3] R.E. Moss, Advanced Molecular Quantum Mechanics, Chapman and Hall, London, 1973.

[4] L.L. Foldy, S.A. Wouthuysen, Phys. Rev. 78 (1950) 29.

[5] M. Douglas, N.M. Kroll, Ann. Phys. (Leipzig) 82 (1974) 89.

[6] B.A. Hess, Phys. Rev. A 32 (1985) 756.

[7] E.v. Lenthe, E.J. Baerends, J.G. Snijders, J. Chem. Phys. 99 (1993) 4597.

[8] S. Faas, J.G. Snijders, J.H.v. Lenthe, E.v. Lenthe, E.J. Baerends, Chem. Phys. Lett. 246 (1995) 632.

[9] R. Samzow, B.A. Hess, G. Jansen, J. Chem. Phys. 96 (1992) 1227.

[10] K.G. Dyall, J. Chem. Phys. 106 (1997) 9618.

[11] T. Nakajima, K. Hirao, J. Chem. Phys. 113 (2000) 7786.

[12] A. Wolf, M. Reiher, B.A. Hess, J. Chem. Phys. 117 (2002) 9215.

[13] M. Barysz, A.J. Sadlej, J. Chem. Phys. 116 (2002) 2696.

[14] C. van Wullen, C. Michauk, J. Chem. Phys. 123 (2005) 204113.

[15] D. Peng, W. Liu, Y. Xiao, L. Cheng, J. Chem. Phys. 127 (2007) 104106.

[16] J. Sikkema, L. Visscher, T. Saue, M. Iliaš, J. Chem. Phys. 131 (2009) 124116.

[17] W. Liu, Mol. Phys. 108 (2010) 1679.

[18] R. Seeger, J.A. Pople, J. Chem. Phys. 66 (1977) 3045.

[19] H. Fukutome, Int. J. Quantum Chem. 20 (1981) 955.

[20] J.-L. Calais, Adv. Quantum Chem. 17 (1985) 225.

[21] R. McWeeny, Methods of Molecular Quantum Mechanics, Academic Press, London, 1989.

[22] P.-O. Löwdin, I. Mayer, Adv. Quantum Chem. 24 (1992) 79.

[23] S. Hammes-Schiffer, H.C. Andersen, J. Chem. Phys. 99 (1993) 1901.

[24] S.K. Wolff, D. Jayatilaka, G.S. Chandler, J. Chem. Phys. 103 (1995) 4562.

[25] J.L. Stuber, J. Paldus, in: E.J. Brändas, E.S. Kryachko (Eds.), Fundamental World of Quantum Chemistry, Kluwer Academic Publishers, Dordrecht, 2003, p. 67.

[26] J.J. Goings, F. Ding, M.J. Frisch, X. Li, J. Chem. Phys. 142 (2015) 154109.

[27] P. Pulay, Chem. Phys. Lett. 73 (1980) 393. 
[28] P. Pulay, J. Comput. Chem. 3 (1982) 556.

[29] K.N. Kudin, G.E. Scuseria, E. Cancès, J. Chem. Phys. 116 (2002) 8255.

[30] X. Hu, W. Yang, J. Chem. Phys. 132 (2010) 054109.

[31] Y.A. Wang, C.Y. Yam, Y.K. Chen, G. Chen, J. Chem. Phys. 134 (2011) 241103.

[32] Y.K. Chen, Y.A. Wang, J. Chem. Theory Comput. 7 (2011) 3045.

[33] D. Sulzer, S. Iuchi, K. Yasuda, Chem. Phys. Lett. 635 (2015) 201.

[34] A.J. Garza, G.E. Scuseria, J. Chem. Phys. 137 (2012) 054110.

[35] D.G. Luenberger, Y. Ye, Linear and Nonlinear Programming, Springer, New York, 2008.

[36] T. Koga, H. Tatewaki, T. Shimazaki, Chem. Phys. Lett. 328 (2000) 473.

[37] Y. Osanai, T. Noro, E. Miyoshi, M. Sekiya, T. Koga, J. Chem. Phys. 120 (2004) 6408.

[38] J.H.v. Lenthe, R. Zwaans, H.J.v. Dam, M.F. Guest, J. Comput. Chem. 27 (2006) 926.

[39] K. Liu, C.G. Ning, Z.H. Luo, L.L. Shi, J.K. Deng, Chem. Phys. Lett. 497 (2010) 229. 


\section{Figure captions}

Figure 1. SCF convergence behavior in GHFcalculations for $\mathrm{W}(\mathrm{CO})_{6}$ with FP, damping, DIIS, EDIIS, and EDIIS+DIIS. The SF result using EDIIS+DIIS is also shown. The converged energydetermined in the SD calculation was -16809.23228 a.u.

Figure 2.SCF convergence behavior in GHF calculations for $\mathrm{Cr}_{3}$ with FP, damping, DIIS, EDIIS, and EDIIS+DIIS. The SF result using EDIIS+DIIS is also shown. The converged energydetermined in the SD calculation was -3148.60769 a.u.

Figure 3.SCF convergence behavior in GHF calculations for $\mathrm{UF}_{4}$ with $\mathrm{FP}$, damping, DIIS, EDIIS, and EDIIS+DIIS. The SF result using EDIIS+DIIS is also shown. The converged energydetermined in the SD calculation was -28380.18867 a.u. 


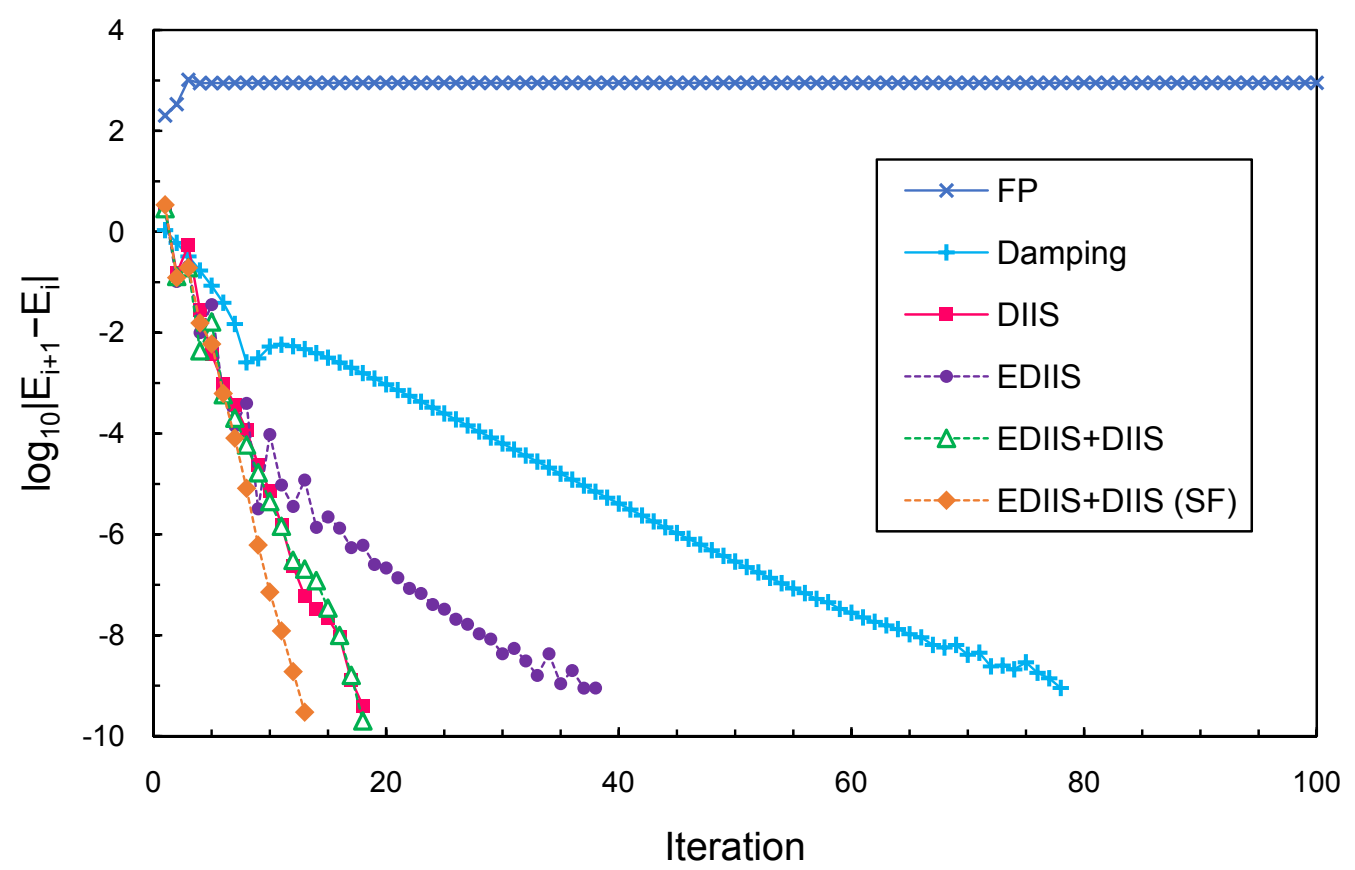

Figure 1 of Nakano et al. 


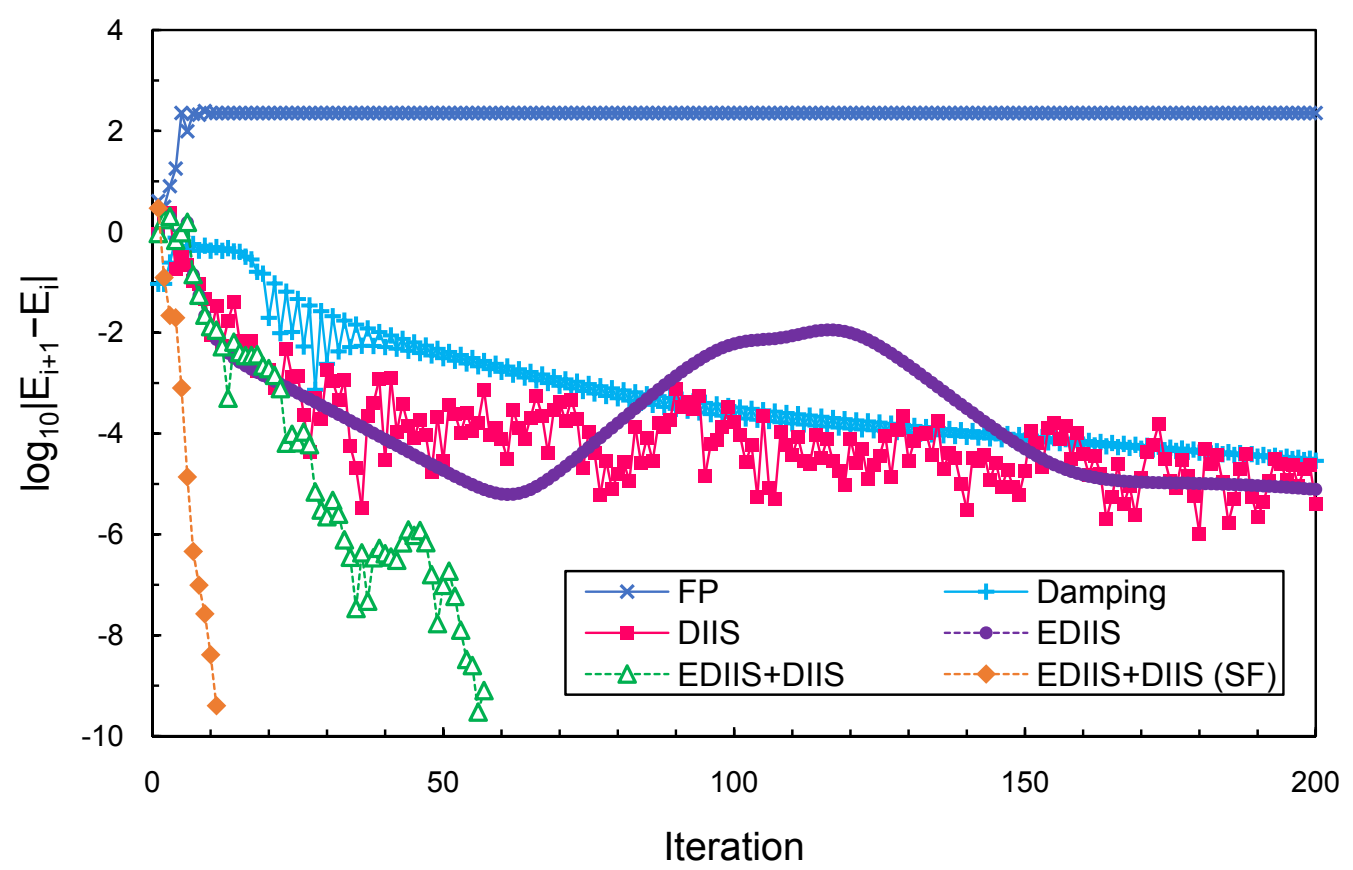

Figure 2 of Nakano et al. 


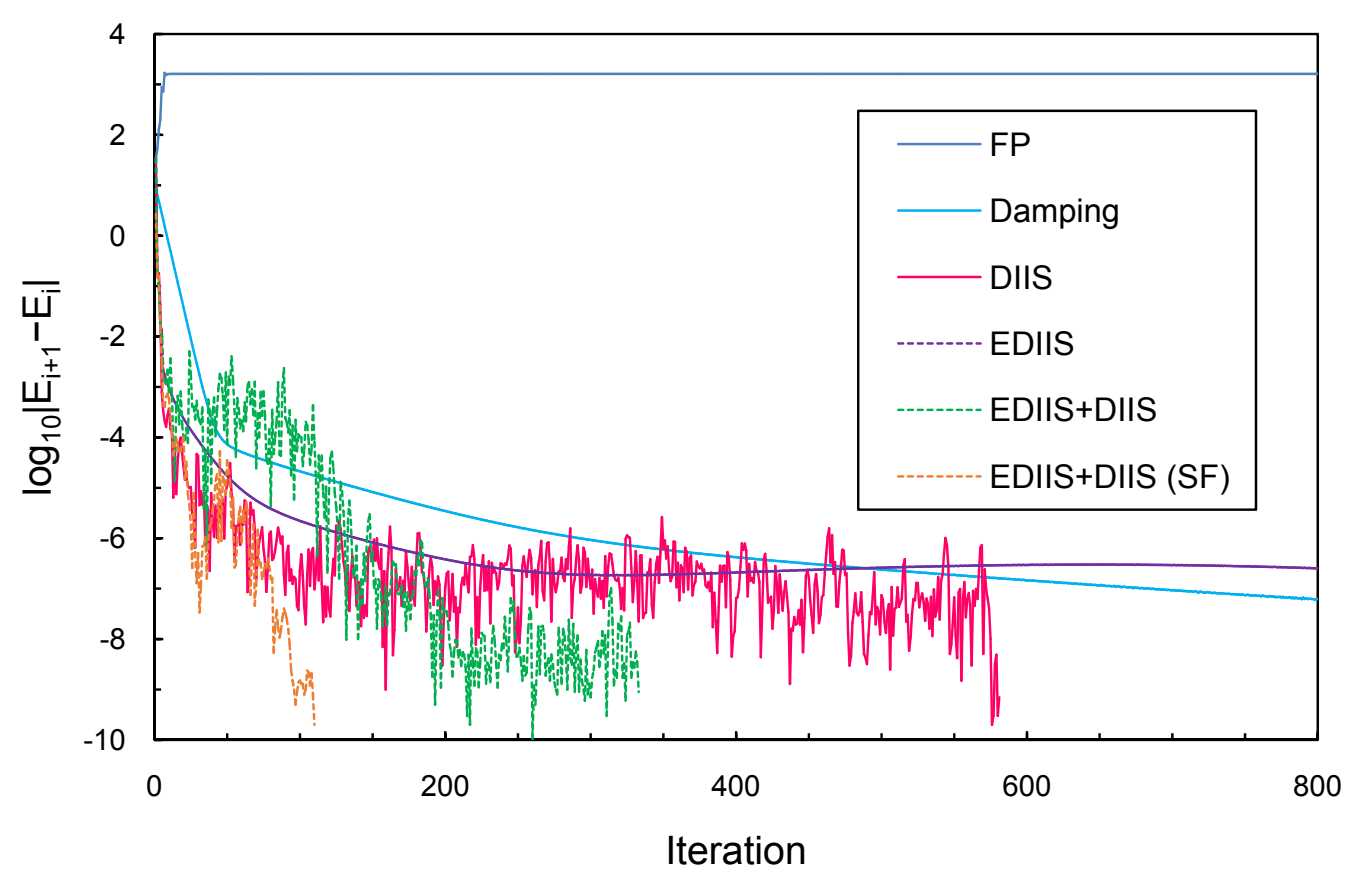

Figure 3 of Nakano et al. 
Table 1. Number of cycles required for SCF convergence in s- and p-block neutral atomic systems at the SF- and SD-IODKH levels using the FP, damping, DIIS, EDIIS, and EDIIS+DIIS algorithms.

\begin{tabular}{|c|c|c|c|c|c|c|c|c|c|c|c|c|}
\hline \multirow{2}{*}{ Element } & \multirow{2}{*}{$Z$} & \multirow{2}{*}{$2 S+1$} & \multicolumn{5}{|c|}{ SF-IODKH } & \multicolumn{5}{|c|}{ SD-IODKH } \\
\hline & & & FP & Damping & DIIS & EDIIS & $\overline{\text { EDIIS+DIIS }}$ & FP & Damping & DIIS & EDIIS & EDIIS+DIIS \\
\hline $\begin{array}{l}\text { Period } 1 \\
\mathrm{He}\end{array}$ & 2 & 1 & 7 & 63 & 6 & 7 & 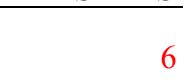 & 7 & 63 & 6 & 7 & - \\
\hline $\begin{array}{l}\text { Period } 2 \\
\mathrm{Li} \\
\mathrm{Be} \\
\mathrm{B} \\
\mathrm{C} \\
\mathrm{N} \\
\mathrm{O} \\
\mathrm{F} \\
\mathrm{Ne}\end{array}$ & $\begin{array}{r}3 \\
4 \\
5 \\
6 \\
7 \\
8 \\
9 \\
10\end{array}$ & $\begin{array}{l}2 \\
1 \\
2 \\
2 \\
3 \\
4 \\
3 \\
2 \\
1\end{array}$ & $\begin{array}{l}17 \\
10 \\
17 \\
14 \\
14 \\
14 \\
17 \\
22\end{array}$ & $\begin{array}{l}69 \\
70 \\
72 \\
76 \\
77 \\
80 \\
80 \\
79\end{array}$ & $\begin{array}{r}9 \\
7 \\
10 \\
10 \\
10 \\
10 \\
11 \\
10\end{array}$ & $\begin{array}{l}17 \\
10 \\
17 \\
13 \\
14 \\
14 \\
16 \\
19\end{array}$ & $\begin{array}{r}9 \\
7 \\
10 \\
10 \\
10 \\
11 \\
12 \\
12\end{array}$ & $\begin{array}{r}17 \\
9 \\
244 \\
333 \\
77 \\
266 \\
17 \\
22\end{array}$ & $\begin{array}{r}69 \\
70 \\
848 \\
\text { N.C. } \\
284 \\
888 \\
80 \\
79\end{array}$ & $\begin{array}{r}10 \\
7 \\
28 \\
25 \\
25 \\
22 \\
11 \\
13\end{array}$ & $\begin{array}{r}17 \\
9 \\
244 \\
300 \\
93 \\
266 \\
16 \\
19\end{array}$ & $\begin{array}{r}10 \\
7 \\
58 \\
24 \\
174 \\
27 \\
12 \\
13\end{array}$ \\
\hline $\begin{array}{l}\text { Period } 3 \\
\mathrm{Na} \\
\mathrm{Mg} \\
\mathrm{Al} \\
\mathrm{Si} \\
\mathrm{P} \\
\mathrm{S} \\
\mathrm{Cl} \\
\mathrm{Ar}\end{array}$ & $\begin{array}{l}11 \\
12 \\
13 \\
14 \\
15 \\
16 \\
17 \\
18\end{array}$ & $\begin{array}{l}2 \\
1 \\
2 \\
3 \\
4 \\
4 \\
3 \\
2 \\
1\end{array}$ & $\begin{array}{l}20 \\
15 \\
19 \\
14 \\
15 \\
16 \\
15 \\
14\end{array}$ & $\begin{array}{l}80 \\
81 \\
83 \\
79 \\
79 \\
81 \\
81 \\
81\end{array}$ & $\begin{array}{r}13 \\
11 \\
11 \\
11 \\
10 \\
11 \\
11 \\
9\end{array}$ & $\begin{array}{l}19 \\
14 \\
18 \\
14 \\
14 \\
16 \\
15 \\
13\end{array}$ & $\begin{array}{l}13 \\
11 \\
11 \\
11 \\
10 \\
11 \\
12 \\
10\end{array}$ & $\begin{array}{r}19 \\
15 \\
142 \\
11 \\
109 \\
267 \\
338 \\
13\end{array}$ & $\begin{array}{r}80 \\
81 \\
494 \\
78 \\
397 \\
892 \\
\text { N.C. } \\
81\end{array}$ & $\begin{array}{r}17 \\
15 \\
25 \\
10 \\
141 \\
33 \\
181 \\
11\end{array}$ & $\begin{array}{r}18 \\
14 \\
142 \\
11 \\
133 \\
269 \\
307 \\
13\end{array}$ & $\begin{array}{r}13 \\
12 \\
23 \\
10 \\
127 \\
32 \\
205 \\
11\end{array}$ \\
\hline $\begin{array}{l}\text { Period } 4 \\
\mathrm{~K} \\
\mathrm{Ca} \\
\mathrm{Ga} \\
\mathrm{Ge} \\
\mathrm{As} \\
\mathrm{Se} \\
\mathrm{Br} \\
\mathrm{Kr}\end{array}$ & $\begin{array}{l}19 \\
20 \\
31 \\
32 \\
33 \\
34 \\
35 \\
36\end{array}$ & $\begin{array}{l}2 \\
1 \\
2 \\
3 \\
4 \\
3 \\
2 \\
2 \\
1\end{array}$ & $\begin{array}{r}19 \\
18 \\
\text { N.C. } \\
\text { N.C. } \\
\text { N.C. } \\
25 \\
22 \\
21\end{array}$ & $\begin{array}{l}83 \\
82 \\
83 \\
81 \\
82 \\
83 \\
84 \\
84\end{array}$ & $\begin{array}{l}14 \\
12 \\
14 \\
12 \\
12 \\
13 \\
12 \\
11\end{array}$ & $\begin{array}{l}21 \\
19 \\
20 \\
19 \\
18 \\
18 \\
18 \\
18\end{array}$ & $\begin{array}{l}13 \\
12 \\
12 \\
13 \\
12 \\
13 \\
13 \\
12\end{array}$ & $\begin{array}{r}19 \\
18 \\
\text { N.C. } \\
\text { N.C. } \\
\text { N.C. } \\
\text { N.C. } \\
285 \\
20\end{array}$ & $\begin{array}{r}82 \\
82 \\
480 \\
81 \\
314 \\
\text { N.C. } \\
\text { N.C. } \\
84\end{array}$ & $\begin{array}{r}75 \\
108 \\
23 \\
19 \\
52 \\
221 \\
86 \\
16\end{array}$ & $\begin{array}{r}20 \\
17 \\
139 \\
19 \\
94 \\
\text { N.C. } \\
538 \\
17\end{array}$ & $\begin{array}{r}14 \\
15 \\
20 \\
14 \\
101 \\
159 \\
34 \\
14\end{array}$ \\
\hline $\begin{array}{l}\text { Period } 5 \\
\mathrm{Rb} \\
\mathrm{Sr} \\
\mathrm{In} \\
\mathrm{Sn} \\
\mathrm{Sb} \\
\mathrm{Te}\end{array}$ & $\begin{array}{l}37 \\
38 \\
49 \\
50 \\
51 \\
52\end{array}$ & $\begin{array}{l}2 \\
1 \\
2 \\
3 \\
4 \\
3\end{array}$ & $\begin{array}{l}25 \\
18 \\
21 \\
19 \\
19 \\
19\end{array}$ & $\begin{array}{l}95 \\
90 \\
84 \\
83 \\
83 \\
83\end{array}$ & $\begin{array}{l}14 \\
12 \\
13 \\
12 \\
12 \\
12\end{array}$ & $\begin{array}{l}22 \\
18 \\
20 \\
18 \\
17 \\
18\end{array}$ & $\begin{array}{l}15 \\
14 \\
13 \\
13 \\
12 \\
13\end{array}$ & $\begin{array}{r}24 \\
18 \\
89 \\
18 \\
79 \\
\text { N.C. }\end{array}$ & $\begin{array}{r}96 \\
101 \\
321 \\
82 \\
304 \\
\text { N.C. }\end{array}$ & $\begin{array}{l}52 \\
58 \\
19 \\
14 \\
25 \\
26\end{array}$ & $\begin{array}{r}22 \\
23 \\
93 \\
16 \\
80 \\
606\end{array}$ & $\begin{array}{l}17 \\
19 \\
18 \\
12 \\
25 \\
29\end{array}$ \\
\hline
\end{tabular}


Table 1. (Continued.)

\begin{tabular}{|c|c|c|c|c|c|c|c|c|c|c|c|c|}
\hline \multirow{2}{*}{ Element } & \multirow{2}{*}{$Z$} & \multirow{2}{*}{$2 S+1$} & \multicolumn{5}{|c|}{ SF-IODKH } & \multicolumn{5}{|c|}{ "SD-IODKH } \\
\hline & & & FP & Damping & DIIS & EDIIS & EDIIS+DIIS & FP & Damping & DIIS & EDIIS & EDIIS+DIIS \\
\hline Period 5 & & & & & & & & & & & & \\
\hline I & 53 & 2 & 18 & 84 & 12 & 17 & 13 & 386 & N.C. & 45 & 254 & 41 \\
\hline $\mathrm{Xe}$ & 54 & 1 & 19 & 84 & 10 & 17 & 11 & 18 & 84 & 11 & 17 & 13 \\
\hline \multicolumn{13}{|l|}{ Period 6} \\
\hline $\mathrm{Cs}$ & 55 & 2 & 23 & 87 & 14 & 22 & 14 & 25 & 84 & 15 & 20 & 14 \\
\hline $\mathrm{Ba}$ & 56 & 1 & 19 & 85 & 16 & 24 & 15 & 18 & 85 & 430 & 33 & 22 \\
\hline $\mathrm{Tl}$ & 81 & 2 & 32 & 87 & 13 & 22 & 15 & 58 & 157 & 19 & 54 & 18 \\
\hline $\mathrm{Pb}$ & 82 & 3 & 26 & 88 & 13 & 20 & 14 & 36 & 88 & 18 & 33 & 17 \\
\hline $\mathrm{Bi}$ & 83 & 4 & 24 & 88 & 13 & 20 & 14 & 78 & 312 & 36 & 93 & 217 \\
\hline Po & 84 & 3 & 23 & 88 & 13 & 20 & 14 & 927 & N.C. & 31 & N.C. & 43 \\
\hline At & 85 & 2 & 23 & 89 & 12 & 20 & 14 & 305 & 921 & 113 & 314 & 43 \\
\hline $\mathrm{Rn}$ & 86 & 1 & 23 & 89 & 11 & 20 & 14 & 22 & 89 & 13 & 19 & 15 \\
\hline \multicolumn{13}{|l|}{ Period 7} \\
\hline Fr & 87 & 2 & 28 & 104 & 15 & 24 & 16 & 26 & 152 & 96 & 23 & 17 \\
\hline $\mathrm{Ra}$ & 88 & 1 & 20 & 97 & 14 & 19 & 15 & 20 & 117 & 19 & 18 & 16 \\
\hline
\end{tabular}

*N.C. means no convergence in 1000 cycles. 
Table 2. Number of cycles required for SCF convergence in d-block neutral atomic systems at the SF- and SD-IODKH levels using the FP, damping, DIIS, EDIIS, and EDIIS+DIIS algorithms.

\begin{tabular}{|c|c|c|c|c|c|c|c|c|c|c|c|c|}
\hline \multirow{2}{*}{ Element } & \multirow{2}{*}{$Z$} & \multirow{2}{*}{$2 S+1$} & \multicolumn{5}{|c|}{ SF-IODKH } & \multicolumn{5}{|c|}{ "SD-IODKH } \\
\hline & & & $\mathrm{FP}$ & Damping & DIIS & EDIIS & EDIIS+DIIS & FP & Damping & DIIS & EDIIS & EDIIS+DIIS \\
\hline \multicolumn{13}{|l|}{ Period 4} \\
\hline $\mathrm{Sc}$ & 21 & 2 & N.C. & N.C. & 24 & 283 & 25 & N.C. & N.C. & 195 & N.C. & 605 \\
\hline $\mathrm{Ti}$ & 22 & 3 & N.C. & N.C. & 26 & 125 & 26 & N.C. & N.C. & 14 & 786 & 192 \\
\hline V & 23 & 4 & N.C. & 281 & 21 & 121 & 27 & N.C. & N.C. & 44 & 838 & 476 \\
\hline $\mathrm{Cr}$ & 24 & 7 & N.C. & 81 & 13 & 22 & 13 & N.C. & 584 & 304 & N.C. & 38 \\
\hline $\mathrm{Mn}$ & 25 & 6 & N.C. & 82 & 15 & 22 & 13 & N.C. & 268 & N.C. & 55 & 22 \\
\hline $\mathrm{Fe}$ & 26 & 5 & N.C. & 88 & 42 & 22 & 14 & N.C. & N.C. & 639 & 27 & 114 \\
\hline Co & 27 & 4 & N.C. & 218 & 23 & 111 & 24 & N.C. & N.C. & N.C. & 856 & 447 \\
\hline $\mathrm{Ni}$ & 28 & 3 & N.C. & 92 & 15 & 35 & 16 & N.C. & N.C. & 28 & N.C. & 464 \\
\hline $\mathrm{Cu}$ & 29 & 2 & N.C. & 87 & 15 & 51 & 17 & N.C. & 87 & 19 & 27 & 16 \\
\hline $\mathrm{Zn}$ & 30 & 1 & N.C. & 84 & 12 & 20 & 12 & N.C. & 84 & 16 & 19 & 25 \\
\hline \multicolumn{13}{|l|}{ Period 5} \\
\hline Y & 39 & 2 & 219 & 710 & 24 & 221 & 30 & 518 & N.C. & 885 & 470 & 195 \\
\hline $\mathrm{Zr}$ & 40 & 3 & 198 & 478 & 29 & 155 & 24 & N.C. & N.C. & 32 & N.C. & 49 \\
\hline $\mathrm{Nb}$ & 41 & 6 & 61 & 79 & 17 & 22 & 14 & 878 & N.C. & N.C. & N.C. & 247 \\
\hline Mo & 42 & 7 & 24 & 80 & 11 & 19 & 12 & 166 & 729 & N.C. & 185 & 34 \\
\hline $\mathrm{Tc}$ & 43 & 6 & 809 & 148 & 26 & 39 & 14 & N.C. & 223 & N.C. & 66 & 50 \\
\hline $\mathrm{Ru}$ & 44 & 5 & 99 & 163 & 19 & 189 & 60 & N.C. & N.C. & 585 & 389 & N.C. \\
\hline $\mathrm{Rh}$ & 45 & 4 & N.C. & 358 & 25 & 108 & 26 & N.C. & N.C. & 470 & N.C. & 142 \\
\hline $\mathrm{Pd}$ & 46 & 1 & N.C. & 159 & 13 & 41 & 13 & N.C. & 284 & 17 & 39 & 16 \\
\hline $\mathrm{Ag}$ & 47 & 2 & N.C. & 112 & 14 & 25 & 14 & N.C. & 242 & 16 & 24 & 15 \\
\hline $\mathrm{Cd}$ & 48 & 1 & N.C. & 84 & 12 & 22 & 13 & N.C. & 84 & 15 & 21 & 14 \\
\hline \multicolumn{13}{|l|}{ Period 6} \\
\hline $\mathrm{Lu}$ & 71 & 2 & N.C. & 469 & 21 & 42 & 19 & N.C. & 959 & 40 & 347 & 555 \\
\hline Hf & 72 & 3 & N.C. & 587 & 27 & 168 & 29 & N.C. & N.C. & 82 & 608 & 447 \\
\hline $\mathrm{Ta}$ & 73 & 4 & N.C. & 288 & 33 & 151 & 38 & N.C. & N.C. & 78 & 296 & 841 \\
\hline W & 74 & 5 & N.C. & 446 & 20 & 80 & 28 & N.C. & 110 & 31 & 226 & 21 \\
\hline $\mathrm{Re}$ & 75 & 6 & N.C. & 213 & 30 & 22 & 15 & N.C. & 261 & 27 & 57 & 26 \\
\hline Os & 76 & 5 & N.C. & 226 & 24 & 43 & 16 & N.C. & 884 & 560 & 179 & 290 \\
\hline $\mathrm{Ir}$ & 77 & 4 & N.C. & 367 & 43 & 144 & 24 & N.C. & N.C. & N.C. & 689 & 239 \\
\hline $\mathrm{Pt}$ & 78 & 3 & N.C. & 409 & 28 & 124 & 22 & N.C. & N.C. & 25 & 984 & 628 \\
\hline $\mathrm{Au}$ & 79 & 2 & N.C. & 94 & 14 & 46 & 18 & N.C. & 89 & 19 & 33 & 18 \\
\hline $\mathrm{Hg}$ & 80 & 1 & N.C. & 88 & 12 & 21 & 13 & N.C. & 87 & 16 & 20 & 14 \\
\hline \multicolumn{13}{|l|}{ Period 7} \\
\hline $\mathrm{Lr}$ & 103 & 2 & N.C. & 516 & 56 & 175 & 26 & N.C. & 266 & 201 & 111 & 37 \\
\hline
\end{tabular}

*N.C. means no convergence in 1000 cycles. 
Table 3. Number of cycles required for SCF convergence in f-block neutral atomic systems at the SF- and SD-IODKH levels using the FP, damping, DIIS, EDIIS, and EDIIS+DIIS algorithms.

\begin{tabular}{|c|c|c|c|c|c|c|c|c|c|c|c|c|}
\hline \multirow{2}{*}{ Element } & \multirow{2}{*}{$Z$} & \multirow{2}{*}{$2 S+1$} & \multicolumn{5}{|c|}{ SF-IODKH } & \multicolumn{5}{|c|}{ "SD-IODKH } \\
\hline & & & $\mathrm{FP}$ & Damping & DIIS & EDIIS & EDIIS+DIIS & FP & Damping & DIIS & EDIIS & EDIIS+DIIS \\
\hline \multicolumn{13}{|l|}{ Period 6} \\
\hline $\mathrm{La}$ & 57 & 2 & N.C. & 733 & N.C. & 177 & 33 & N.C. & N.C. & 275 & 437 & N.C. \\
\hline $\mathrm{Ce}$ & 58 & 1 & N.C. & 262 & 30 & N.C. & 26 & N.C. & N.C. & N.C. & N.C. & 338 \\
\hline $\mathrm{Pr}$ & 59 & 4 & N.C. & N.C. & 157 & 430 & 61 & N.C. & N.C. & 801 & N.C. & 221 \\
\hline $\mathrm{Nd}$ & 60 & 5 & N.C. & N.C. & 110 & 438 & 161 & N.C. & N.C. & N.C. & N.C. & N.C. \\
\hline $\mathrm{Pm}$ & 61 & 6 & N.C. & N.C. & 47 & 679 & 64 & N.C. & 515 & 286 & N.C. & 227 \\
\hline $\mathrm{Sm}$ & 62 & 7 & N.C. & N.C. & 71 & 685 & 31 & N.C. & 951 & 486 & 258 & 33 \\
\hline $\mathrm{Eu}$ & 63 & 8 & N.C. & 223 & 132 & 74 & 29 & N.C. & 257 & N.C. & 95 & 48 \\
\hline Gd & 64 & 9 & N.C. & 819 & 29 & 203 & 31 & N.C. & N.C. & 711 & 890 & N.C. \\
\hline $\mathrm{Tb}$ & 65 & 6 & N.C. & 688 & 33 & N.C. & N.C. & N.C. & 878 & 366 & 334 & 58 \\
\hline Dy & 66 & 5 & N.C. & N.C. & N.C. & 304 & 464 & N.C. & N.C. & 488 & N.C. & 123 \\
\hline Но & 67 & 4 & N.C. & N.C. & 69 & 387 & 104 & N.C. & 204 & 554 & 94 & 28 \\
\hline $\mathrm{Er}$ & 68 & 3 & N.C. & N.C. & N.C. & 291 & 34 & N.C. & N.C. & N.C. & 813 & N.C. \\
\hline $\mathrm{Tm}$ & 69 & 2 & N.C. & N.C. & N.C. & 467 & 35 & N.C. & N.C. & 699 & 948 & 478 \\
\hline $\mathrm{Yb}$ & 70 & 1 & N.C. & 94 & 20 & 30 & 17 & N.C. & 108 & N.C. & 32 & 28 \\
\hline \multicolumn{13}{|l|}{ Period 7} \\
\hline Ac & 89 & 2 & N.C. & 907 & 23 & 72 & 25 & N.C. & N.C. & 149 & 155 & 43 \\
\hline Th & 90 & 3 & 348 & 191 & 32 & 240 & 42 & N.C. & N.C. & 32 & N.C. & 35 \\
\hline $\mathrm{Pa}$ & 91 & 4 & N.C. & N.C. & N.C. & 892 & 124 & N.C. & N.C. & N.C. & 937 & N.C. \\
\hline $\mathrm{U}$ & 92 & 5 & N.C. & N.C. & N.C. & N.C. & 470 & N.C. & N.C. & N.C. & 423 & 70 \\
\hline $\mathrm{Np}$ & 93 & 6 & N.C. & N.C. & 82 & N.C. & 407 & N.C. & N.C. & 54 & 380 & N.C. \\
\hline $\mathrm{Pu}$ & 94 & 7 & N.C. & N.C. & 30 & 465 & 36 & N.C. & N.C. & 35 & 292 & N.C. \\
\hline $\mathrm{Am}$ & 95 & 8 & N.C. & 269 & 27 & 805 & 579 & N.C. & 305 & 368 & 93 & 55 \\
\hline $\mathrm{Cm}$ & 96 & 9 & N.C. & 726 & 24 & 150 & 41 & N.C. & 816 & 458 & 204 & 429 \\
\hline $\mathrm{Bk}$ & 97 & 6 & N.C. & N.C. & 51 & N.C. & 75 & N.C. & 580 & N.C. & 268 & 180 \\
\hline $\mathrm{Cf}$ & 98 & 5 & N.C. & N.C. & N.C. & 245 & 557 & N.C. & N.C. & N.C. & 884 & 468 \\
\hline Es & 99 & 4 & N.C. & N.C. & N.C. & 107 & 24 & N.C. & N.C. & 309 & 888 & 595 \\
\hline $\mathrm{Fm}$ & 100 & 3 & N.C. & N.C. & 22 & 50 & 20 & N.C. & N.C. & 434 & 642 & 498 \\
\hline Md & 101 & 2 & N.C. & 153 & 23 & 548 & 34 & N.C. & N.C. & N.C. & 101 & 39 \\
\hline No & 102 & 1 & N.C. & 94 & 18 & 35 & 16 & N.C. & 165 & N.C. & 94 & 61 \\
\hline
\end{tabular}

*N.C. means no convergence in 1000 cycles. 
Table 4. Summary of the SCF convergence behaviors in s/p-, d-, and f-block neutral atomic systems at the SF- and SD-IODKH levels using the FP, damping, DIIS, EDIIS, and EDIIS+DIIS algorithms.

\begin{tabular}{|c|c|c|c|c|c|c|c|c|c|c|c|}
\hline \multirow{2}{*}{ System } & \multirow{2}{*}{ Characteristic } & \multicolumn{5}{|c|}{ SF-IODKH } & \multicolumn{5}{|c|}{ SD-IODKH } \\
\hline & & FP & Damping & DIIS & EDIIS & EDIIS+DIIS & FP & Damping & DIIS & EDIIS & EDIIS+DIIS \\
\hline s/p-block & \#Failure & 3 & 0 & 0 & 0 & 0 & 5 & 7 & 0 & 2 & 0 \\
\hline \multirow[t]{3}{*}{ elements } & $\%$ Average & 56.4 & 578.3 & -4.6 & 44.6 & - & 187.3 & 493.4 & 28.2 & 169.0 & - \\
\hline & $\%$ Best & 16.7 & 950.0 & 0.0 & 16.7 & - & 16.7 & 950.0 & 0.0 & 16.7 & - \\
\hline & $\%$ Worst & 100.0 & 550.0 & 0.0 & 50.0 & - & 327.2 & 324.4 & 98.2 & 179.3 & - \\
\hline d-block & \#Failure & 25 & 2 & 0 & 0 & 0 & 28 & 15 & 6 & 6 & 1 \\
\hline \multirow[t]{3}{*}{ elements } & $\%$ Average & 1012.2 & 1056.6 & 7.5 & 307.5 & - & 148.8 & 56.6 & -16.7 & 40.6 & - \\
\hline & $\%$ Best & 100.0 & 558.3 & -8.3 & 58.3 & - & 1085.7 & 500.0 & 0.0 & 35.7 & - \\
\hline & $\%$ Worst & 1248.3 & 1083.3 & -6.7 & 371.7 & - & 4.4 & 14.0 & 5.2 & 17.0 & - \\
\hline f-block & \#Failure & 27 & 16 & 8 & 5 & 1 & 28 & 18 & 11 & 6 & 7 \\
\hline \multirow[t]{3}{*}{ elements } & $\%$ Average & 165.4 & 227.9 & -60.7 & 157.8 & - & - & 147.5 & 98.2 & 118.0 & - \\
\hline & $\%$ Best & 2075.0 & 487.5 & 12.5 & 87.5 & - & - & 285.7 & 14.3 & 14.3 & - \\
\hline & $\%$ Worst & -39.9 & 56.6 & -72.9 & 54.1 & - & - & 59.8 & 34.6 & 59.3 & - \\
\hline
\end{tabular}

*\#Failure represents the number of elements which failed the SCF convergence within 1000 cycles.

** $\% \mathrm{X}$ means the ratioof $\mathrm{X}$ to EDIIS+DIIS, i.e., $\% \mathrm{X}=(\mathrm{X}-\mathrm{X}($ EDIIS+DIIS $)) / \mathrm{X}(\mathrm{EDIIS}+\mathrm{DIIS}) \times 100 \%$. 
